(C) Springer-Verlag 1992

\title{
Erratum
}

\section{Allelic diversity at the primate major histocompatibility complex DRB6 locus}

\author{
Alfredo Corell, Pablo Morales, Pilar Varela, Estela Paz-Artal, J. Manuel Martin-Villa, Jorge Martinez-Laso, \\ Antonio Arnaiz-Villena
}

Immunogenetics 36: 33-38, 1992

The nomenclature in Figures 1 and 2B is erroneous due to a printing error, the correct figures are shown below. Also, the first sentence of the note added in proof should read: "Recently we have obtained another $H L A-D R B 6$ allele sequence linked to DRw16 individuals: $D R B 6^{*} 0202$ "; and the first sentence of the second paragraph should read: "The names $D R B 6^{*} 0101, D R B 6^{*} 0201$, and $D R B 6^{*} 0202 \ldots . "$

\section{DRB-Amp A}

DR B - 600

$\begin{array}{lllllllllllllllllllllllllllllllllllllll}3 & 4 & 5 & 6 & 7 & 8 & 9 & 10 & 11 & 12 & 23 & 14 & 15 & 16 & 17 & 18 & 19 & 20 & 21 & 22 & 23 & 24 & 25 & 26 & 27 & 28 & 29 & 30 & 31 & 32 & 33 & 34 & 35 & 36 & 37 & 38 & & & \end{array}$ HLA-DRE $* 0101$ CCC ACA GCA CGT TTC TTG GAG CAG GCT AAG TGT GAG TG̈T CAT ATC TTC AAT GGG ATG AAG CGG GTG CAG TAC CTG AAC AGA TAC ATC CAT AAA CGG GAG GAG AAC CTG $\begin{array}{rl}{ }_{H} & * 0201 \\ \text { HLA-DRBVI } & \ldots\end{array}$ PatI-DRB6 $\$ 0301$ a

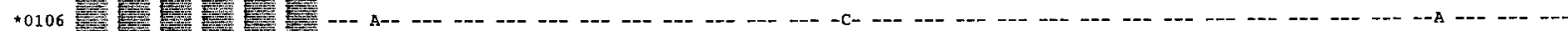
$\$ 0107$ d $\star 0108$ v Gogo-DRB6 0203 was

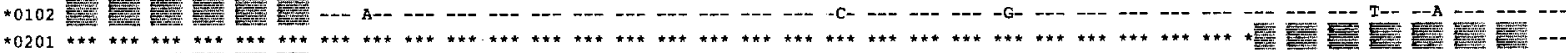

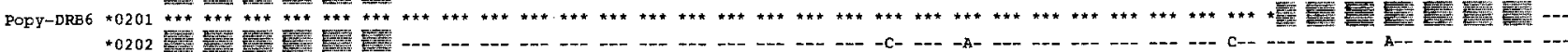

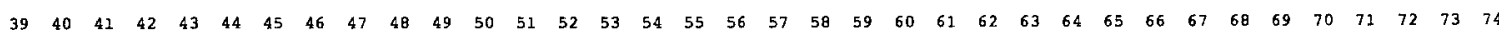
HLA-DRB6 *0101 CCC TTC GAC AGC GAC GTG GAG GAG TTC CAG GCA GTT ACG GAA CTG GGG CGG CCT GTC GCA GAG AAC TGG AAC AGC CAG AAG GGA ATC CTG GAG GAG AAT CGG GAC AAG

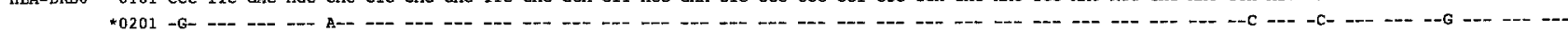

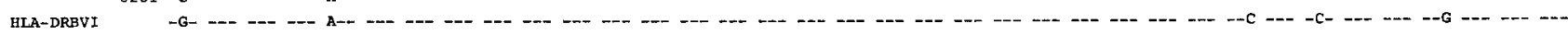
Patr-DREG *0301 -G- --T -.- --- -..

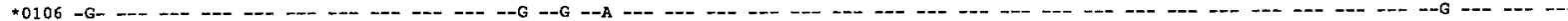

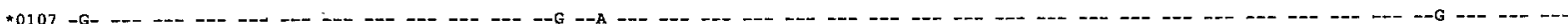

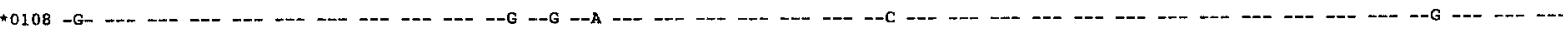

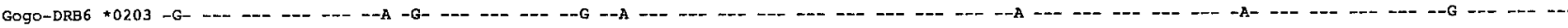

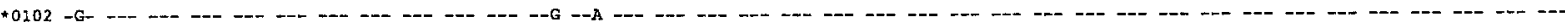

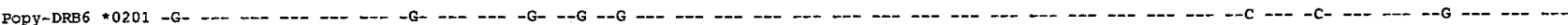

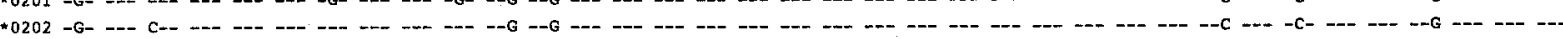

\section{DRB - Amp B}

DRB - 6125

$\begin{array}{llllllllllllllllllllll}75 & 76 & 77 & 78 & 79 & 80 & 81 & 82 & 83 & 84 & 85 & 86 & 87 & 88 & 89 & 90 & 91 & 92 & 93 & 94\end{array}$

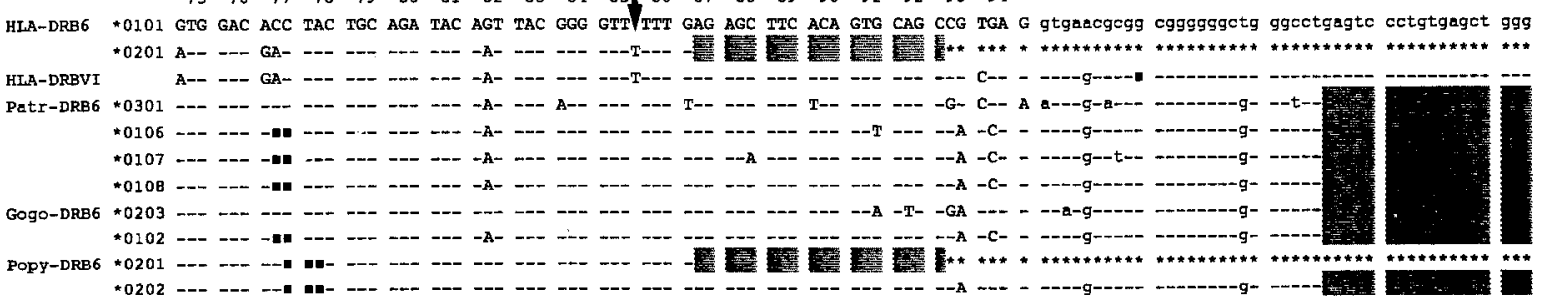

Fig. 1. Comparison of HLA-DRB6, Patr-DRB6, Gogo-DRB6, and Popy-DRB6 exon-2 and intron-2 beginning nucleotide sequences. Uppercase and lowercase letters denote exon and intron nucleotides, respectively. Asterisks represent nonsequenced regions, black squares represent deletions. The insertion is depicted by a thin vertical arrow and shadowed areas correspond to the primers used for amplification in each sequence. Thick black horizontal arrows show length, direction, sequence, and name of used primers. $H L A-D R B 6^{*} 0101$ and $* 0201$ are from Corell and co-workers 1991; HLA-DRBVI is from Figueroa and co-workers 1991. EMBL accession numbers: 1) HLA-DRB6*0101, X53357, *0201: X53358; 2) PatrDRB6*0301, X60798,*0106, X60799, *0107: X60800,*0108, X60801; 3) Gogo-DRB6*0203, X60802,*0102, X60803; 4) Popy-DRB6*0201, X60804, *0202: X60805. Registration dates: a) human alleles: August 16, 1990; b) ape alleles: July 9, 1991. 


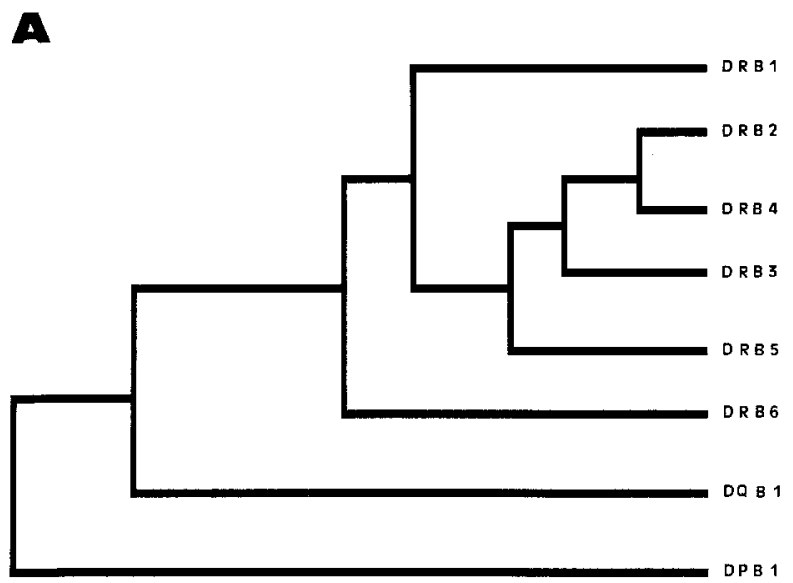

$\mathbf{B}$

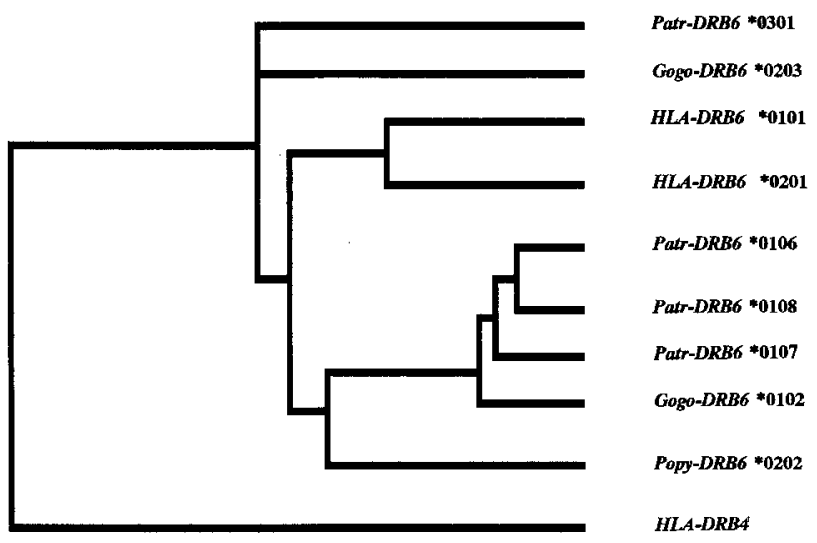

Fig. 2. A Dendrogram depicting the relationship between human $D R B$ consensus sequences for exon- 2 comparisons. B Dendrogram depicting the relationship between nine $D R B 6$ alleles from four different primate species. 